\title{
A survey of Health Professionals' views on acceptable gestational age and termination of pregnancy for fetal anomaly
}

Lisa Crowe, ${ }^{1}$ Ruth H Graham, ${ }^{2}$ Stephen C Robson, ${ }^{3}$ Judith Rankin. ${ }^{1}$

${ }^{1}$ Institute of Health \& Society, Baddiley-Clark Building, Newcastle University, NE2 4AX.

${ }^{2}$ School of Geography, Politics and Sociology, $5^{\text {th }}$ Floor Claremont Bridge Building, Newcastle University, NE1 7RU.

${ }^{3}$ Institute of Cellular Medicine, $3{ }^{\text {rd }}$ Floor William Leech Building, Newcastle University, NE2 $4 \mathrm{HH}$.

Corresponding Author:

Lisa Crowe

Institute of Health \& Society, Baddiley-Clark Building, Newcastle University, NE2 4AX, UK. (+44) 01912083812

Lisa.Crowe@ncl.ac.uk 


\begin{abstract}
Termination of pregnancy for fetal anomaly is legal in the UK with no upper limit, if two doctors, in good faith, agree "there is a substantial risk that if the child were born it would suffer from such physical or mental abnormalities as to be seriously handicapped". This is Clause E of the Human Fertlisation and Embryology Act. The most commonly sighted Clause is C, which states "the pregnancy has not exceeded its twenty-fourth week and that the continuance of the pregnancy would involve risk, greater than if the pregnancy were terminated, of injury to the physical or mental health of the pregnant woman". This study aimed to investigate health professionals' views on gestational age and acceptable termination of pregnancy for fetal anomaly (TOPFA).
\end{abstract}

We undertook a questionnaire survey of UK health professionals working in fetal medicine, obstetrics and gynaecology and neonatology. A study pack consisting of a self-completion questionnaire, an invitation letter, participant information sheet, and a stamped addressed return envelope, were sent to health professionals. We used four fetal anomalies as case study examples in the questionnaire: isolated cleft lip, hypoplastic left heart, spina bifida and trisomy 21 . These anomalies were chosen as they differed in terms of the type of anomaly, the type of impairment, and the perceived severity.

Forty-one study packs were returned. For anomalies deemed less serious, later gestational ages were an important consideration when deciding acceptable TOPFA. Long term prognosis was considered an important factor in deciding whether a TOPFA was acceptable alongside gestational age. Clause $\mathrm{C}$ of the current UK legislation, which allows a legal termination prior to 24 weeks gestational age if continuing with the pregnancy would impact the mental health of the mother, was deemed a reasonable option for termination when parents are requesting a TOPFA. 
For each case study example, health professionals responded that TOPFA at ' 25 weeks and over' was acceptable (cleft lip $n=1$; hypoplastic left heart $n=19$; spina bifida $n=13$ and Trisomy 21 $\mathrm{n}=10)$. Professionals also distinguished between their personal and professional views.

These findings offer new insight into how gestational age considerations influence professionals' conceptualisation of acceptable TOPFA.

\section{Key words}

Termination of pregnancy, termination of pregnancy for fetal anomaly, gestational age, health professionals.

\section{Abbreviations}

TOP - Termination of pregnancy

TOPFA - Termination of pregnancy for fetal anomaly

HFEA - Human Fertilisation and Embryology Act

HLH - Hypoplastic left heart

NHS - National Health Service

RCOG - Royal College of Obstetrics and Gynecology

FASP - Fetal Anomaly Screening Programme

\section{Acknowledgements}

We would like to thank all the study participants for giving up their time to complete the questionnaire.

\section{Funding Sources}

This research was funded by a UK Medical Research Council/Economic and Social Research Council Integrated studentship, G0800128-3/1. 


\section{Main text}

In England and Wales, the Abortion Act(1) and the Human Fertilisation and Embryology Act (HFEA)(2) allow termination of pregnancy for fetal anomaly (TOPFA) at any gestation. Clause E of the HFEA states that a TOPFA is legal if two doctors, in good faith, agree "there is a substantial risk that if the child were born it would suffer from such physical or mental abnormalities as to be seriously handicapped"(2). There is no list that defines which fetal anomalies meet these criteria. However some TOPFA is performed under Clause $\mathrm{C}$ of the HFEA which states that: 'the pregnancy has not exceeded its twenty-fourth week and that the continuance of the pregnancy would involve risk, greater than if the pregnancy were terminated, of injury to the physical or mental health of the pregnant woman.' In this circumstance it is presumed the doctors do not believe the anomaly is sufficiently serious to fulfil the criteria for Clause E.

The Royal College of Obstetrics and Gynaecology $(3,4)($ RCOG) has produced guidelines to support professionals when making such decisions. The National Health Service (NHS) Fetal Anomaly Screening Programme (FASP) defines a fetal anomaly as an abnormality which "may indicate the baby might die shortly after birth, conditions that may benefit from treatment before birth, to plan delivery in an appropriate hospital/Centre and/or to optimize treatment after the baby is born"(5). All routine antenatal ultrasound screening for fetal anomalies is recommended between $18^{+0}$ and $20^{+6}$ weeks of pregnancy, and is offered to all pregnant women through the NHS FASP programme.(5)

Fetal viability is defined as the point where the fetus can survive independently of the pregnant woman. Viability is considered to be 24 weeks' gestational age in the UK.(6) The RCOG define a 'threshold of viability' as $23^{+0}$ weeks to $24^{+6}$ weeks' gestational age.(7) These guidelines state an 
international consensus that "at 22 weeks of gestation there is no hope of survival, and that up to $22^{+6}$ weeks is considered to be the cut-off of human viability and for week $25^{+0}$ onwards there is also a general agreement that active management should be offered".(7) While survival following premature birth has increased, morbidity has not,(7) and preterm labour, in particular prior to 26 weeks' gestation, continues to be a primary cause of neonatal mortality and morbidity.(8) Active intervention after the birth of extremely premature infants has been shown to vary across Europe.(8) This was particularly the case for births prior to 25 weeks' gestation.(8) While viability is considered to be around 24 weeks' gestational age, the Clauses in the UK HFEA ${ }^{1}$ do not specify viability, only gestational age. Viability, however, was a consideration setting the limits in the TOP legislation.(9) Fetal viability has also been used in further debates in 2008 around reducing the upper limit to 22 or 20 weeks, although no changes to legislation occurred.(10) In 2016, there were 180,794 terminations conducted under Clause C representing $97 \%$ of the total.(11) That same year, there were reported to be 3,208 terminations conducted under Clause E; this represents $2 \%$ of the total number of terminations.(11) Research has found that medical professionals raise concerns about the difficulty of defining terms such as 'substantial' and 'serious'.(12-14) This is coupled with variations in views on impairment and disability among parents and medical providers,(8) which may both impact perceptions of 'substantial' and 'serious'. Using Clause C, after a diagnosis of fetal anomaly, is an option available to medical professionals if the pregnancy is sub 24 weeks' gestation. Using Clause $\mathrm{C}$ in the provision of a termination, removes the fetal anomaly as the primary reason for the termination.(15) This allows medical professionals to authorise a termination request by parents, that they may not feel falls into Clause E, or where they feel additional moral arguments are legitimate to the decision making process.(15) So while the legislation offers the legal framework for TOPFA, this does

\footnotetext{
${ }^{1}$ Abortion in Northern Ireland remains governed by the Offenses Against the Persons Act, 1861.
} 
not mean that all TOP with an affected fetus is captured in the data for terminations under Clause E.

This study aimed to investigate health professionals' views on gestational age and acceptable TOPFA using four fetal anomalies as case study examples.

\section{Sample Characteristics}

The sample consisted of medical professionals at consultant level $(\mathrm{n}=41 / 114$, response rate $36 \%$ ) from a range of specialties; obstetrics, gynaecology, neonatology, paediatrics and fetal medicine in four NHS sites in the North East of England. Each of the four NHS sites had a primary investigator affiliated to this study. These primary investigators acted as a gatekeeper to the medical professional community, distributing the research packs to the relevant professionals at that particular research site.

\section{Fetal anomaly subtypes used in the case studies}

Four fetal anomaly subtypes were selected for inclusion in the study. The cases were; isolated cleft lip, hypoplastic left heart (HLH), spina bifida and Trisomy 21. These anomalies were selected based on the type of anomaly (structural or chromosomal), the type of impairment (physical or cognitive), and the perceived severity. Within the case studies, the prognoses ranged from lethal without medical intervention (HLH) to a perceived normal life experience following intervention (isolated cleft lip). The range of anomaly characteristics included allowed us to explore the impact of such variations in professionals' understandings of how gestational age impacts on their decisions about TOPFA. 


\section{Methods}

This research forms part of a larger mixed methods study investigating TOP for non-lethal fetal anomaly.(16) A study pack consisting of a self-completion questionnaire, which included fixed response and open ended questions, an invitation letter, participant information sheet, and a stamped addressed return envelope, were sent to medical professionals. The questionnaire was split into 4 sections: the first asked some general questions about TOP; section 2 were case study specific questions; section 3 some demographic information; and section 4 was an invitation to interview. This paper is focusing on data gained from section 2 , the case study specific questions, which asked questions specific to gestational age. In the questionnaire, participants were asked a specific question about the gestational age at which they thought TOPFA became acceptable for each of the four case study examples. Respondents were provided with six categories of gestational age to choose from, with space to comment on their response. These categories were; never; 25 or more weeks; 22-24 weeks; 19-21 weeks; 13-18 weeks; less than 12 weeks. Illustrative quotes are presented with the results. Eight health professionals did not select a specific gestational age category but used the 'comments' section to provide an answer $(n=7)$ in which gestational age was given.

\section{Results}

A total of 114 study packs were sent out and 41 were returned (response rate $36 \%$ ). There were $22(53.6 \%)$ female and $19(46.3 \%)$ male health professionals in the study sample. Additional information on the sample characteristics cannot be provided due to confidentiality and the sensitivity of the research topic. The gestational age categories at which respondents considered TOPFA was acceptable for each anomaly case study are presented in Figure 1.

\section{Cleft lip}


Almost half of the respondents $(20 ; 48.8 \%)$ responded that TOPFA for cleft lip was not acceptable at any gestational age. Health professionals provided some insight into their choices which included; personal opinion statements, some explanation regarding treatment and distinguishing between personal and professional opinions.

Personally never, professionally $<20$ weeks.

I can't even imagine why someone would terminate a potentially bealthy baby for a cleft lip and it certainly shouldn't even be offered as an option.

Cleft lip is very treatable, so I see no reason for TOP.

However, seven (17.1\%) respondents considered that TOPFA was acceptable at 22-24 weeks of gestation (Fig. 1). Rather than the anomaly being the primary justification of TOPFA, health professionals appear to adopt Clause $\mathrm{C}$ of the legislation.

Never: because you TOP for the maternal mental health Clause $C$ not for Clause E.

... both professionally and personally I wouldn't 'recommend/support' a TOP request for isolated cleft lip. However, if after counselling by appropriate professionals, the patient was still very distressed requesting TOP, I would proceed but under Clause $C$.

One health professional commented on a personal experience of performing a TOPFA for cleft lip;

I have been coerced into TOP (mid trimester 20/40) for isolated cleft lip many years ago. I regret it. 


\section{Hypoplastic left heart}

Nineteen (43\%) respondents said they would support TOPFA at any gestational age (Fig. 1). The open responses suggested that this choice was based on health professionals' knowledge regarding the difficulty of diagnosing HLH prenatally.

Difficult diagnosis before 20 week anomaly ultrasound.

I don't think definitive diagnosis made locally prior to 19 weeks gestation.

The perceived severity of the condition was also discussed by respondents, who were thus employing the 'no upper limit' aspect of Clause E.

... at any stage, when the diagnosis is certain.

HLH is a grim condition therefore TOP I believe would be acceptable. Children with HLH have a great deal of suffering.

I feel personally uncomfortable about late terminations and would accept if a serious medical condition e.g. HLH.

Respondents also suggested that alternatives to TOPFA should be considered depending on the severity of the prognosis, and the availability of treatment.

If prognosis is not bad, surgical correction often first should be offered.

$H L H$, though may require several surgeries, is treatable. 
Differences between a personal and professional opinions were also noted.

Not an easy question, personally $<24$ weeks, professionally $>31$ weeks.

\section{Spina bifida}

The majority of respondents selected two gestational age categories as being acceptable for TOPFA for spina bifida: 22-24 weeks $(14 ; 34.1 \%)$ and 25 weeks and over $(13 ; 31.7 \%)$. Health professionals indicated that the severity of the anomaly (in terms of type and extent of the defect) was the basis for the differences in views around acceptability of TOPFA at different gestational ages.

Before viability, if a minor defect.

Depends upon level of lesion.

Again, dependent on the degree of spina bifida and progression of associated issues (such as bydrocephalus), I would deem this to be a 'serious' anomaly. Would seek tertiary opinion $>24$ weeks.

It should also depend on what kind of spina bifida. Some are asymptomatic and hence no need for TOP because they would live normal life.

Respondents also considered later gestational age when discussing the severity and thus acceptability of TOPFA for spina bifida. 
I feel personally uncomfortable about late terminations unless serious medical condition spina bifida as a diagnosis covers a range of severities.

I doubt isolated spina bifida would be diagnosed prior to 15 weeks locally. I feel anxious about saying that TOP for isolated spina bifida beyond 30 weeks is acceptable especially if diagnosis made at 20 weeks.

Late diagnosis and 'likely' (up arrow) bandicap could persuade me that later TOP would be acceptable.

As with isolated cleft lip, one respondent distinguished between their personal and professional opinions.

Same as for HLH - Not an easy question, personally $<24$ weeks, professionally $>31$ weeks.

\section{Trisomy 21}

Sixteen (39\%) and 10 (24.4\%) respondents selected '22-24 weeks' and '25 weeks and over' respectively. The responses to open questions indicated that views were influenced by the presence or absence of other structural anomalies.

TOP only if multiple defects associated with the chromosomal abnormality.

This is presuming that diagnosis made before 25 weeks and that the fetus does not have any known structural abnormality (e.g. AVSD etc).

Depends when diagnosed, but more difficult to justify in 'uncomplicated' cases $>24$ weeks. 
Would ask for tertiary opinion.

Any gestation if other anomalies e.g. cardiac abnormalities.

Respondents commonly noted that they were personally against TOP for Trisomy 21 but could appreciate the request in a professional capacity.

Feel personally uncomfortable unless serious medical problems. Should terminate Downs syndrome earlier but justifying of additional problems later may affect need for late termination.

Professional opinion. Not personal.

I wouldn't agree personally but professionally I understand why, for a lot of parents, continuing with a Downs pregnancy is not right for them.

I would not recommend this TOP for Downs syndrome but if it in parental choice I will understand and accept this.

I personally feel TOP for Downs is never acceptable. However if current ethics allows TOP then it must be done $<21 / 40$.

\section{Discussion}

This study investigated health professionals' views on the influence of gestational age on the acceptability of TOPFA using four different fetal anomalies as case study examples. Our findings suggest a number of factors influence health professionals' decision-making about gestational age and acceptable TOPFA. These include: the prognosis of an anomaly; the option of invoking 
Clause $\mathrm{C}$ of the HFEA; and differentiating between personal and professional opinions.

Professionals have the option of offering a TOP under Clause $C$ if the pregnancy is sub-24 weeks, and they do not feel the anomaly fulfilled the criteria for Clause E. Given the likelihood of health professionals being able to separate their personal beliefs from their professional role is limited,(17) it is of vital importance to consider these issues. A range of views both within and between case studies, and perspectives of health professionals who are working in this field, were provided. This range is of particular interest considering that the four case studies covered both an anomaly considered to be minor and one that is more serious in terms of functionality.

Gestational age is often considered in conjunction with the long term prognosis of the fetal anomaly. For example, tension emerged when discussing spina bifida and Trisomy 21 and acceptable TOPFA. Acceptable TOPFA was negotiated at later gestational ages if there was the presence of additional anomalies which may influence the long term prognosis of the affected fetus. Clause $\mathrm{C}$ was revealed to be an option health professionals adopt after a diagnosis of fetal anomaly. This was in instances where health professionals did not agree that the fetal anomaly in question was serious enough to justify a TOPFA under Clause E of the HFEA. The acceptability of terminations around 22-24 weeks, and the use of Clause C, may suggest that the age of viability is being adopted as an indicator of acceptable TOP. However, only one health professional explicitly referred to viability in their decision making; this was in reference to spina bifida. While the option of Clause $\mathrm{C}$ may be preferred by health professionals who may have ethical concerns about the reason for the termination request, there is no research into parents' feelings in this distinction. This is of particular interest as parents terminating a wanted child do not wish to be likened to those who are terminating an unwanted pregnancy(18). A further important consideration is that professional views have the potential of resulting in no access to termination services after 24 weeks, after the point at which Clause $\mathrm{C}$ can be used. 
Health professionals indicated that they considered TOPFA was acceptable at less than 19 weeks gestational age. Unlike Trisomy 21, where screening is offered at 11-14 weeks in the UK, screening for structural fetal anomalies with ultrasound is not offered until $18^{+0}$ and $20^{+6}$ weeks pregnancy.(4) Thus, in principle, TOP for structural anomalies is not a consideration prior to 18 weeks. This did not, however, stop professionals selecting earlier gestation categories. While the structural anomalies chosen can be reliably diagnosed after 12 weeks (19-23), responses may be suggestive of a more idealised model of care or what health professionals think may be achievable diagnoses if accessing care at specialised Centre's in a timely manner. This is of importance as gestational age appears to influence decisions about acceptable TOPFA. This may subsequently influence attitudes towards TOPFA requests and the counselling parents receive after a diagnosis.

The data also reveal other factors that inform the decision making process. Some differences between personal and professional opinions were reported, with health professionals often being more accepting of later TOPFA in a professional capacity than in their personal views. However, some health professionals reported that they do not differentiate between TOP at any gestational age. This may explain why the ' 25 weeks and over' category was an option selected for all four case studies in at least some instances. Other research has found the personal views of health professionals may influence counselling after a diagnosis of fetal anomalies; for example, religious affiliation.(24) This insight is important given the high sensitivity of late TOP and the potential impact of professional views on the patient. These issues are further intensified when considering that the wordings of the relevant legislation, both in the UK and other countries that provide TOPFA, are open to interpretation,(25) thus potentially creating subjective decision making. Health professionals in the field are in a unique position where they may be very influential to patients' decision making during a very vulnerable time. For example, research has found parents feeling counselled towards TOPFA after a diagnosis of fetal anomaly.(26, 27) 
Thus, it is essential that the potential impact of professionals' personal views on issues surrounding gestational age to be considered in discussions of policy making and implementation. This is not to suggest that professionals would be acting with any intentionality, rather that personal views can potentially impact on professional activity in an unconscious manner.(28)

Gestational age has been found in other studies to be an influential factor in issues surrounding TOPFA. Gestational age restrictions have been found to be imposed by healthcare professionals on TOPFA for 'commonly lethal' anomalies (for example, Trisomy 13 and 18).(29) Other research found difficulties accessing a late TOPFA for a lethal anomaly after 24 weeks in the US.(30) The 'age of fetus' has also been found to be influential when deciding whether to undertake a surgical TOP.(31) Relating specifically to fetal anomaly, research has found differing levels of objection to TOP depending on the reason behind the request (for sex selection, $82 \%$ of the sample of obstetricians and gynaecologists, objected to TOP, whereas for cardiopulmonary disease, 16\% objected).(32) For UK medical students, willingness to sign the paperwork to authorise TOP decreased with late pregnancy compared to early pregnancy.(33) Increasing gestational age has also been revealed as an indicator of reduced willingness to participate in both TOP and TOPFA services.(34) Harris et al. 2011, however noted a power imbalance in these decision making processes given it is the health professionals who are determining whether a reason is good enough to justify TOP,(32) highlighting the importance of understanding health professionals' views as they can be seen to be a gatekeeper to services. It should be noted that a number of these studies have been undertaken in the USA, where the debates and legislation on both TOP and TOPFA procedures differ to that of England and Wales, but the fact that these debates extend beyond the UK suggests that these issues are of broader relevance. 
The study has several strengths. While the sample is not extensive, we have found important data pertaining to gestational age and acceptable TOPFA. We have also included different health professional groups, and a set of four case study examples that encompass a range of anomaly characteristics. The data also demonstrate some professionals' differences between their personal and professional opinions. This may suggest professionals' ability and willingness to work within a professional paradigm despite it being in conflict with their own personal opinions.

However, the study also has limitations. There is a high number of non-responses. While it is important not to detract from the valuable insights provided by the 41 respondents, we must acknowledge that if the response rate had been higher, the results may have been different. The data were derived from a survey with fixed choice answers and space for additional comments. This may have resulted in ambiguities if respondents were not able to fully express their reasons. Our question offered no definition of severity of the fetal anomaly which also may have impacted responses. It is important to note that there is a considerable range of disability, with an uncertain prognosis within some of these case studies. The question this paper poses to professionals did not provide respondents with opportunities to comment in detail on the complexity of the characteristics case study examples.

Our study has specifically asked health professionals to select a gestational age range by anomaly subtype. The findings therefore add to existing knowledge on provision of TOPFA by offering an insight into the limits in provision to which professionals will go to under Clause E. We also provide an insight into some of the reasoning behind the boundaries identified by professionals, often demonstrating their differing personal and professional limits to acceptability in TOPFA provision. In our small sample, the category ' 25 weeks and over' remained a legitimate termination option for some professionals in all the case study examples provided. This suggests a range of perspectives continue to work within the health care setting, and additional research is 
needed to understand and further analyse these competing views. Only then can we assess the long term impact this may have on patient choice.

\author{
References
}

1. Abortion Act. Abortion Act, 19671967 [cited 2014 14th July]. Available from: http://www.legislation.gov.uk/ukpga/1967/87/pdfs/ukpga_19670087_en.pdf.

2. Human Fertilisation and Embryology Act. Human Fertilisation and Embryology Act 2008: Legislation.gov.uk; 2008 [cited 2014 25th January]. Available from: http://www.legislation.gov.uk/ukpga/2008/22/contents.

3. Royal College of Obstetricians and Gynaecologists. Termination of pregnancy for fetal abnormality in England, Wales and Scotland. Report of a Working Party. London: 1996. 4. Royal College of Obstetricians and Gynaecologists. Termination of pregnancy for fetal abnormality in England, Scotland and Wales. Report of a Working Party. London: 2010. 5. Fetal Anomaly Screening Programme. 2015 [8th January, 2018]. Available from: https://www.gov.uk/government/uploads/system/uploads/attachment_data/file/421650/FASP _Standards_April_2015_final_2_.pdf.

6. Parliament Select Committee. Select Committee on Science and Technology, Twelfth Report. 2007.

7. Royal College of Obstetrics and Gynecology. Perinatal management of pregnant women at the threshold of infant viability (The obstetric perspective). 2014.

8. Gallagher K, Martin J, Keller M, Marlow N. European variation in decision-making and parental involvement during preterm birth. Archives of Disease in Childhood-Fetal and Neonatal Edition. 2014:fetalneonatal-2013-305191.

9. Ingham R, Lee E, Clements SJ, Stone N. Reasons for second trimester abortions in England and Wales. Reproductive health matters. 2008;16(31):18-29.

10. Draper ES, Alfirevic Z, Stacey F, Hennessy E, Costeloe K. An investigation into the reporting and management of late terminations of pregnancy (between $22+0$ and 26+ 6 weeks of gestation) within NHS Hospitals in England in 2006: the EPICure preterm cohort study. BJOG: An International Journal of Obstetrics \& Gynaecology. 2012;119(6):710-5. 11. Department of Health. Abortion Statistics, England and Wales: 2016. 2017. Available from:

https://www.gov.uk/government/uploads/system/uploads/attachment_data/file/618533/Aborti on_stats_2016_commentary_with_tables.pdf.

12. Lotto R, Smith LK, Armstrong N. Clinicians' perspectives of parental decisionmaking following diagnosis of a severe congenital anomaly: a qualitative study. BMJ open. 2017;7(5):e014716.

13. Fisher J, Lafarge C. Women's experience of care when undergoing termination of pregnancy for fetal anomaly in England. Journal of Reproductive and Infant Psychology. 2015;33(1):69-87. 
14. Savulescu J. Is current practice around late termination of pregnancy eugenic and discriminatory? Maternal interests and abortion. Journal of Medical Ethics. 2001;27(3):16571.

15. Crowe L, Graham R, Robson S, Rankin J. Negotiating acceptable termination of pregnancy for non-lethal fetal anomaly: A qualitative study of professional perspectives. BMJ Open. 2018;8(3).

16. Crowe L. Termination of pregnancy for non-lethal fetal anomalies: Professional perspectives: Newcastle University; 2014.

17. Lipp A. A review of termination of pregnancy: prevalent health care professional attitudes and ways of influencing them. Journal of Clinical Nursing. 2008;17(13):1683-8. 18. Lafarge C, Mitchell K, Fox P. Termination of pregnancy for fetal abnormality: a meta-ethnography of women's experiences. Reproductive health matters. 2014;22(44):191201.

19. Brătilă E, Andreea I, Monica M. Early ultrasound evaluation for fetal cardiovascular congenital abnormalities. 2016; 12(46): 190-197.

20. Sepulveda W, Wong AE, Sepulveda F, Alcalde JL, Devoto JC, Otayza F. Prenatal diagnosis of spina bifida: from intracranial translucency to intrauterine surgery. Child's Nervous System. 2017;33(7):1083-99.

21. Orlandi E, Rossi C, Perino A, Cucinella G, Orlandi F. Prospective sonographic detection of spina bifida at 11-14 weeks and systematic literature review. The Journal of Maternal-Fetal \& Neonatal Medicine. 2016;29(14):2363-7.

22. Syngelaki A, Chelemen T, Dagklis T, Allan L, Nicolaides KH. Challenges in the diagnosis of fetal non - chromosomal abnormalities at 11-13 weeks. Prenatal diagnosis. 2011;31(1):90-102.

23. Chaoui R, Orosz G, Heling KS, Sarut-Lopez A, Nicolaides KH. Maxillary gap at 1113 weeks' gestation: marker of cleft lip and palate. Ultrasound in Obstetrics \& Gynecology. 2015;46(6):665-9.

24. Curlin FA, Lawrence RE, Chin MH, Lantos JD. Religion, Conscience, and Controversial Clinical Practices. New England Journal of Medicine. 2007;356(6):593-600.

25. Dommergues M, Mandelbrot L, Mahieu-Caputo D, Boudjema N, Durand-Zaleski I. Termination of pregnancy following prenatal diagnosis in France: how severe are the foetal anomalies? Prenatal Diagnosis. 2010;30(6):531-9.

26. Guon J, Wilfond BS, Farlow B, Brazg T, Janvier A. Our children are not a diagnosis: the experience of parents who continue their pregnancy after a prenatal diagnosis of trisomy 13 or 18. American Journal of Medical Genetics Part A. 2014;164(2):308-18.

27. Walker LV, Miller VJ, Dalton VK. The health-care experiences of families given the prenatal diagnosis of trisomy 18. Journal of Perinatology. 2008;28(1):12.

28. Kapur N. Unconscious bias harms patients and staff. BMJ. 2015;351.

29. Heuser CC, Eller AG, Byrne JL. Survey of physicians' approach to severe fetal anomalies. Journal of medical ethics. 2012;38(7):391-5.

30. Jacobs AR, Dean G, Wasenda EJ, Porsch LM, Moshier EL, Luthy DA, et al. Late termination of pregnancy for lethal fetal anomalies: a national survey of maternal-fetal medicine specialists. Contraception. 2015;91(1):12-8.

31. Aiyer AN, Ruiz G, Steinman A, Ho GYF. Influence of physician attitudes on willingness to perform abortion. Obstetrics \& Gynecology. 1999;93(4):576-80.

32. Harris LH, Cooper A, Rasinski KA, Curlin FA, Lyerly AD. Obstetriciangynecologists' objections to and willingness to help patients obtain an abortion. Obstetrics and gynecology. 2011;118(4):905.

33. Gleeson R, Forde E, Bates E, Powell S, Eadon-Jones E, Draper H. Medical students' attitudes towards abortion: a UK study. Journal of medical ethics. 2008;34(11):783-7. 
34. Fischer RL, Schaeffer K, Hunter RL. Attitudes of obstetrics and gynecology residents toward abortion participation: a Philadelphia area survey. Contraception. 2005;72(3):200-5. 


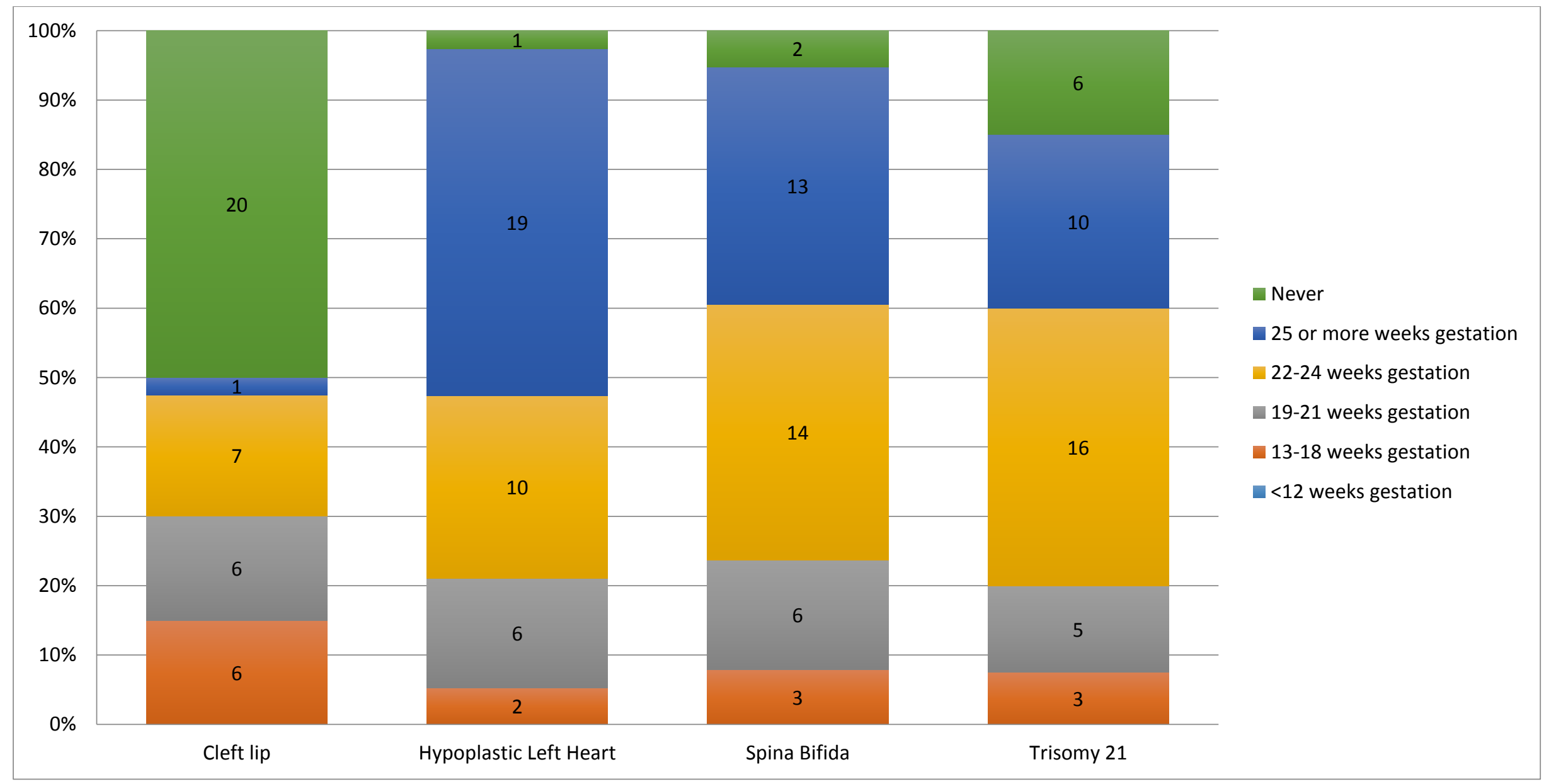

Figure 1: Health professionals' views of the acceptable gestational limit for termination of pregnancy for fetal anomaly. 